\title{
Article
}

Subscriber access provided by Universitätsbibliothek Bern

\section{Donor-Acceptor Properties of a Single-Molecule Altered by On-Surface Complex Formation}

Tobias Meier, Rémy Pawlak, Shigeki Kawai, Yan Geng, Xunshan Liu, Silvio Decurtins, Prokop Hapala, Alexis Baratoff, Shi-Xia Liu, Pavel Jelinek, Ernst Meyer, and Thilo Glatzel

ACS Nano, Just Accepted Manuscript • DOI: 10.1021/acsnano.7b03954 • Publication Date (Web): 21 Jul 2017

Downloaded from http://pubs.acs.org on July 25, 2017

\section{Just Accepted}

"Just Accepted" manuscripts have been peer-reviewed and accepted for publication. They are posted online prior to technical editing, formatting for publication and author proofing. The American Chemical Society provides "Just Accepted" as a free service to the research community to expedite the dissemination of scientific material as soon as possible after acceptance. "Just Accepted" manuscripts appear in full in PDF format accompanied by an HTML abstract. "Just Accepted" manuscripts have been fully peer reviewed, but should not be considered the official version of record. They are accessible to all readers and citable by the Digital Object Identifier (DOI®). "Just Accepted" is an optional service offered to authors. Therefore, the "Just Accepted" Web site may not include all articles that will be published in the journal. After a manuscript is technically edited and formatted, it will be removed from the "Just Accepted" Web site and published as an ASAP article. Note that technical editing may introduce minor changes to the manuscript text and/or graphics which could affect content, and all legal disclaimers and ethical guidelines that apply to the journal pertain. ACS cannot be held responsible for errors or consequences arising from the use of information contained in these "Just Accepted" manuscripts. 


\title{
Donor-Acceptor Properties of a Single-Molecule Altered by On-Surface Complex Formation
}

\author{
Tobias Meier, ${ }^{* \dagger}$ Rémy Pawlak, ${ }^{\dagger}$ Shigeki Kawai, ${ }^{\ddagger}$ Yan Geng, "Xunshan Liu, \\ Silvio Decurtins, "Prokop Hapala, ${ }^{\S}$ Alexis Baratoff, ${ }^{\dagger}$ Shi-Xia Liu, " Pavel \\ Jelínek, ${ }^{\S}$ Ernst Meyer, ${ }^{\dagger}$ and Thilo Glatzel ${ }^{*, \dagger}$ \\ $\dagger$ Department of Physics, University of Basel, Klingelbergstr. 82, 4056 Basel, Switzerland. \\ $\ddagger$ International Center for Materials Nanoarchitectonics, National Institute for Materials \\ Science, 1-1, Namiki, Tsukuba, Ibaraki 305-0044, Japan. \\ PRESTO, Japan Science and Technology Agency, 4-1-8 Honcho, Kawaguchi, Saitama \\ 332-0012, Japan. \\ IDepartment of Chemistry and Biochemistry, University of Bern, Freiestr. 3, 3012 Bern, \\ Switzerland, \\ §Institute of Physics, Academy of Sciences of the Czech Republic, v.v.i., Cukrovarnická 10, \\ 162 00 Prague, Czech Republic. \\ E-mail: tobias.meier@unibas.ch; thilo.glatzel@unibas.ch
}

\begin{abstract}
Electron donor-acceptor molecules are of outstanding interest in molecular electronics and organic solar cells for their intramolecular charge transfer controlled via electrical or optical excitation. The preservation of their electronic character in the ground state upon adsorption on a surface is cardinal for their implementation in such singlemolecule devices. Here, we investigate by atomic force microscopy and scanning tunneling microscopy a prototypical system consisting of a $\pi$-conjugated tetrathiafulvalene-
\end{abstract}


fused dipyridophenazine molecule adsorbed on thin $\mathrm{NaCl}$ films on $\mathrm{Cu}(111)$. Depending on the adsorption site, the molecule is either found in a nearly undisturbed, free state or in a bound state. In the latter case the molecule adopts a specific adsorption site leading to the formation of a chelate complex with a single $\mathrm{Na}^{+}$alkali cation pulled out from the insulating film. Although expected to be electronically decoupled, the charge distribution of the complex is drastically modified leading to the loss of the intrinsic donor-acceptor character. The chelate complex formation is reversible with respect to lateral manipulations enabling tunable donor-acceptor molecular switches activated by on-surface coordination.

Keywords: atomic force microscopy, scanning tunneling microscopy, fused donoracceptor molecules, local contact potential difference, single-molecule, force spectroscopy, coordination.

Electron Donor-Acceptor (DA) molecules are highly regarded in nanoscience for their properties at the base of a emerging class of single molecule based electronic devices. ${ }^{1-5}$ DA molecules with spatially separated electron donating and accepting moieties allow a controlled internal charge transfer. ${ }^{6}$ The spatial separation of the highest occupied molecular orbital (HOMO) and the lowest unoccupied molecular orbital (LUMO) allows excited electron-hole pairs to be split upon photon absorption, thus making DA molecules useful for the conversion of light into electrical current leading to applications in organic solar cells. ${ }^{7}$ Furthermore, electrons can be tuned to resonantly tunnel in and out of the molecule by applying suitable bias voltages. Since there is a preferential direction for electron tunneling through the molecule, DA molecules can also rectify the current and act as molecular diodes. ${ }^{8,9}$ The spatial separation of the electronic orbitals as well as the charge distribution in the ground state determine the properties of DA molecules upon excitation. The question whether these particular properties of DA molecules are still preserved upon adsorption on a surface is cardinal for their integration in molecular electronics. 
At the molecular level, subtle molecule-surface interactions may induce several stable adsorption configurations which depend on the alignment of the molecular structure with respect to the underlying substrate. Using high spatial resolution of non-contact atomic force microscopy (AFM) and scanning tunneling microscopy (STM), the interplay between structural and electronic properties of molecules as well as the visualization of on-surface chemical processes can be elucidated down to the atomic scale. ${ }^{10-17}$ Recently, the small fused DA molecule, TTF-PYZ 2 , was reported to adopt two conformational states on thin $\mathrm{NaCl}$ films on $\mathrm{Cu}(111) .{ }^{10}$ By mapping the local contact potential difference (LCPD), the charge distribution of single molecules ${ }^{10,18}$ was found to be similar to the gas phase, thus demonstrating electronic decoupling from the metallic substrate. ${ }^{11,19-21}$ Pioneering studies with individual atoms have further demonstrated the capability of charging on $\mathrm{NaCl}$ films. ${ }^{22-24}$ The charge state was shown to be related with the adsorption position on the $\mathrm{NaCl}$ lattice which also induced the structural relaxation of the underlying atomic lattice. ${ }^{24,25}$ In contrast, site-dependent charge states were not reported for molecules on thin insulating films since the molecules are usually weakly adsorbed through van der Waals interactions. However, the intrinsic DA character of the molecules implies the coexistence of electron excess and depletion regions at the intramolecular level. Such particular charge distribution may locally interact with the alternating charges of the $\mathrm{NaCl}$ film like in the charged adatom case if those regions are sufficiently localized.

Here, we report the investigation of the charge states of a prototypical DA molecule, the tetrathiafulvalene-fused dipyridophenazine (TTF-dppz) (Fig. 1a), ${ }^{1}$ with respect to its adsorption site on a $\mathrm{NaCl}$ thin film. Using combined STM and high-resolution AFM measurements, we show that in the favored configuration oriented along the apolar axis of $\mathrm{NaCl}$ ([001]) the intramolecular charge distribution changes compared to the other adsorption configurations. This electronic peculiarity results from the local formation of a chelate complex between the dipyridophenazine (dppz) end of the molecule and a single $\mathrm{Na}^{+}$cation pulled out from the $\mathrm{NaCl}$ film. As a result of the chelate complex formation, the intramolecular 
charge distribution is dramatically altered, as revealed by force spectroscopy and density functional theory calculations, which leads to the loss of the intrinsic DA character defined by the spatial distribution of the HOMO localized on a donor and the LUMO on an acceptor in the ground state. ${ }^{10}$ Through lateral molecule-manipulations with the tip, complex formation can be suppressed by varying the adsorption position, which leads to the intrinsic DA character of the molecule. These results underline the fundamental role of atomic-scale coordination to control the electronic properties of donor-acceptor molecules at surfaces.

\section{RESULTS AND DISCUSSION}

To determine the adsorption geometries of the deposited molecules, we conducted highresolution AFM measurements at $5 \mathrm{~K}$ with functionalized CO tips of the TTF-dppz as well as of the $\mathrm{NaCl}$ thin film. ${ }^{26}$ The constant height AFM images reveal two main molecule appearances (Figs. 1b and c) which we name bound and free molecules, respectively. Statistically, we observed a ratio of approximately 2:1 between bound and free molecules upon adsorption at low temperature (Fig. S1). For the bound configuration, the two pyridyl rings are slightly bent downwards as shown by the darker contrast in this area. Between the TTF and the adjacent phenyl ring, the more positive contrast in the frequency shift (Fig. 1b)shows that the molecule is at this position slightly closer to the tip than above the pyridyl units. At the onset of the TTF unit, a bright stripe and two bright spots corresponding to stronger repulsive forces are visible which coincide with the sulfur atom position of the TTF (Fig. 1b). The TTF-dppz molecule image suggests a downwards bending of the dppz unit towards the surface with a maximum height at the edge of the TTF unit resulting in a bridge-shape geometry. Comparing the bound (Fig. 1b) to the free configuration (Fig. 1c), two main differences are observed. First, the contrast along the molecular axis shows a rather flat profile compared to the bridge-shape of the bound configuration. Moreover, the two last 


\section{a}

Acceptor

Donor<smiles></smiles>

b

bound

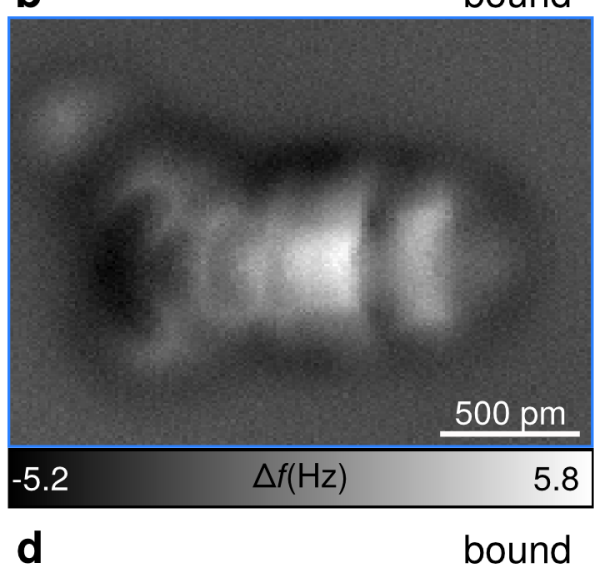

c

free
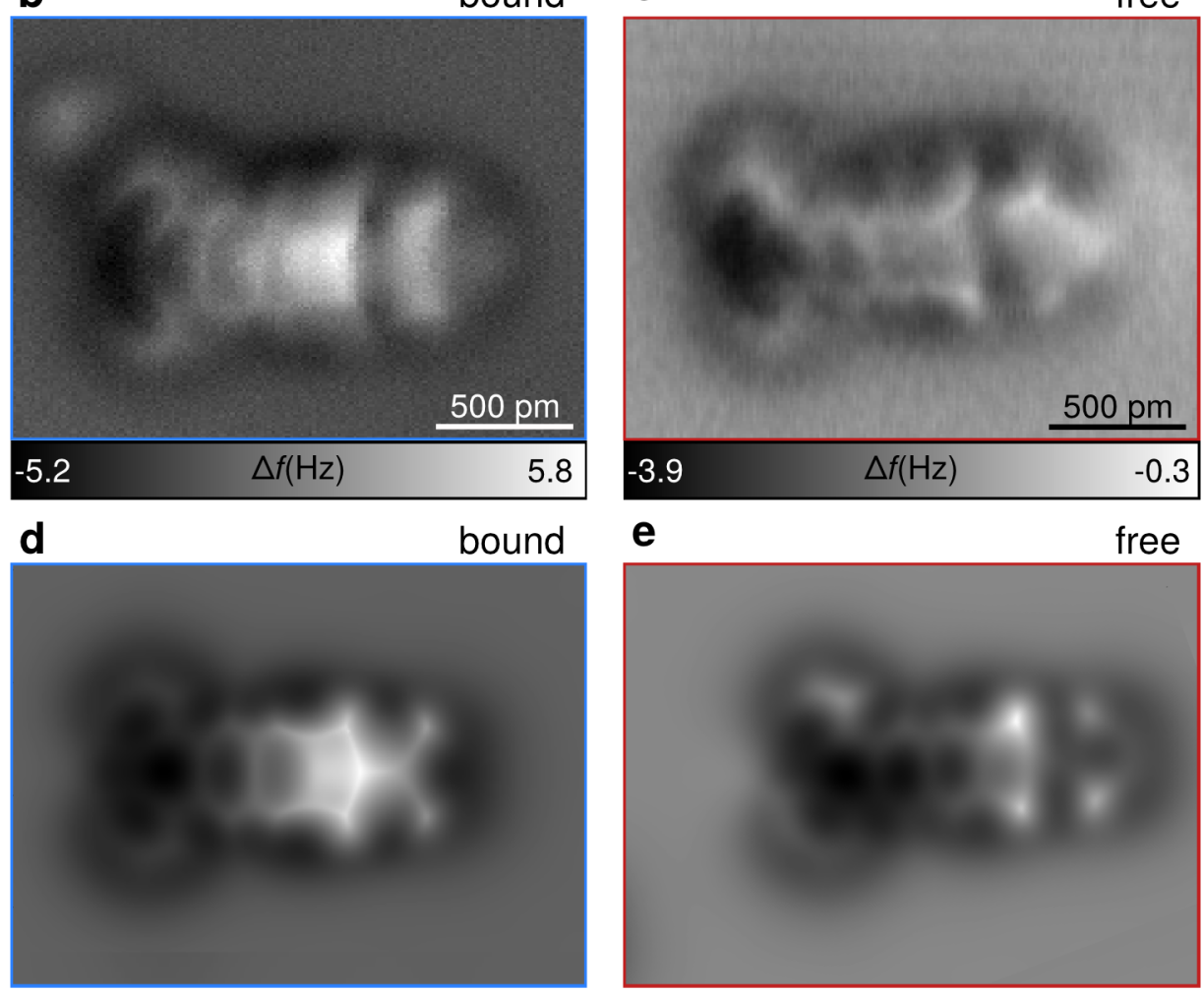

e

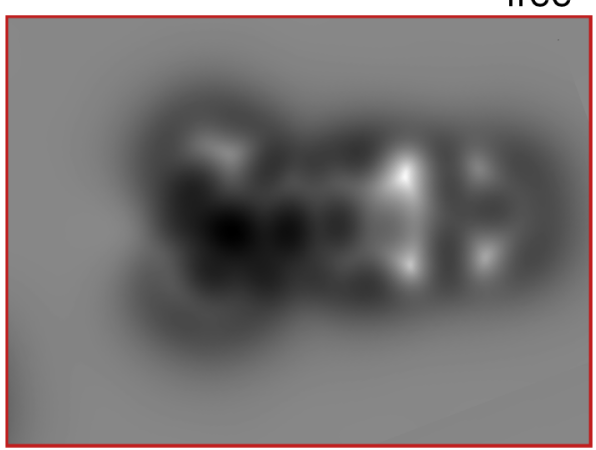

Figure 1: Structure and AFM images of TTF-dppz. a, Chemical structure of TTFdppz. b,c, Constant height $\Delta f$ images of TTF-dppz of bound type at $z=1.27 \AA$ (b) and free type at $z=0.65 \AA$ (c). The heights are referenced to the STM setpoint on the $\mathrm{NaCl}$ layer at a sample bias of $V_{\mathrm{b}}=0.3 \mathrm{~V}, I_{\mathrm{t}}=3 \mathrm{pA}$. The AFM images were recorded on $\mathrm{NaCl}(2 \mathrm{ML}) / \mathrm{Cu}(111)$ at $5 \mathrm{~K}$ with a $\mathrm{CO}$ terminated tip at $V_{\mathrm{b}}=0 \mathrm{~V}$. At the top left in $\mathbf{b}$ a $\mathrm{CO}$ molecule is adsorbed nearby. d,e, Corresponding simulated AFM images calculated with the probe-particle model. 
sulfur atoms of the TTF are slightly tilted for the free molecule. Figures 1d and e show simulated AFM images using the probe-particle model described in ref. ${ }^{27}$. In this model a probe-particle, which represents a CO molecule, is connected to a tip with a spring and is used to determine the interaction between tip and sample in AFM measurements, assuming a specific molecular adsorption geometry calculated by Density Functional Theory (DFT) beforehand. The simulation of the bound configuration (Fig. 1d) reproduces well the bridgeshape of the AFM image (Fig. 1b) as well as the characteristic bright contrast at the TTF moiety. However, the deformation of the phenyl ring and the adjacent part of the TTF is not visible. In the simulated AFM image the molecule stays in its relaxed adsorption geometry and does not have any flexibility which, presumably, plays a role in the contrast of the AFM experiments. The free configuration simulation (Fig. 1e) resembles the AFM features of Fig. 1c such as the relative flat geometry of the molecule and the slight bending of the dppz unit. The distortion of TTF sulfur atoms is however less pronounced compared to the AFM image.

Figure 2 shows the relaxed molecule geometries with respect to their adsorption sites obtained by DFT calculations and in agreement with the determined adsorption geometries by AFM (Fig. S2). ${ }^{26}$ The bound molecule is always adsorbed along the apolar axis of $\mathrm{NaCl}$ (Fig. 2a) whereas three nearly energetically equivalent configurations of the free molecule are found less frequently (free1, free2, free3). These diverse adsorption configurations also explain the slight discrepancy between calculated and experimental AFM images (Figs. 1c and e). The bound molecule (Fig. 2b) has however a characteristic geometry with a height maximum at the TTF onset with a height difference of $\approx 27 \mathrm{pm}$ to the dppz unit pointing towards the surface (Figs. 1b and Fig. 5c). Interestingly, the side-view of Fig. 2b shows a $\mathrm{Na}^{+}$cation pulled out from the $\mathrm{NaCl}$ substrate located below the two nitrogen atoms of the dppz unit. Note, that the $\mathrm{Na}^{+}$cation is part of the $\mathrm{NaCl}$ layer and relatively far away from the tip, so that it cannot be directly detected in the AFM measurements without disturbing the molecule. This adsorption peculiarity also accounts for a significant decrease of the 
molecule adsorption energy of $\approx 200 \mathrm{meV}$ with respect to all free configurations (Fig. $2 \mathrm{~d}$ ). This energetically most favorable configuration is indeed the most frequently observed in the experiments (approximately $70 \%$ of the observed molecules, Fig. S1). In the case of the free configurations (Fig. 2a), the molecule is always slightly rotated compared to the bound configuration. As a result, the adsorption geometry is flatter (Fig. 2c) than the bridge-shape geometry (Fig. 2b) and no pulled out $\mathrm{Na}^{+}$cation is observed. Further rotated adsorption configurations (free2 and free3, Fig. 2a) aligned along the polar $\mathrm{Cl}$ axis are also found and show similar geometries as the free1 one. All orientations misaligned with respect to the apolar $\mathrm{NaCl}$ axis, differ from the bound molecule by the absence of a $\mathrm{Na}^{+}$below the two nitrogens of the dppz unit and are equivalent in terms of geometries and adsorption energies (Fig. 2d) as the free 1 configuration. The sulfur atoms are preferentially located close to $\mathrm{Na}^{+}$ cations. In the case of free1, one of the sulfur atoms is above a $\mathrm{Cl}^{-}$which leads to a higher adsorption height at this position. This observation also holds for the two sulfur atoms closer to the center in the bound configuration. There, the bright stripe occurs because the sulfur atoms stick out. For free2, no tilt or sticking out of the sulfur atoms is observed (Fig. S2e) since the sulfur atoms are symmetrically aligned with the $\mathrm{Na}^{+}$cations underneath. Free3 shows again a slight tilt because of one sulfur atom lying above a $\mathrm{Cl}^{-}$. As a conclusion we see also a contribution of the sulfur atoms to the geometrical adaptations of the molecules with respect to the adsorption site.

To study the impact of those configurations on the intrinsic DA character of the TTFdppz, we performed scanning tunneling spectroscopy (STS) combined with STM (Fig. 3) and resolved the corresponding spatial contributions of HOMO and LUMO of the adsorbed molecule. ${ }^{28}$ The bound molecule shows resonant tunneling at $-2.3 \mathrm{~V}$ (Fig. 3a) where the spatial distribution of the HOMO dominates the STM image (Fig. 3c). The HOMO is clearly located at the donor TTF and only a small tail is observed at the acceptor site. Moreover, the measured HOMO image matches reasonably the density distribution of the HOMO of the gas phase molecule calculated by DFT (Fig. S3c). The LUMO resonance of the bound 
a

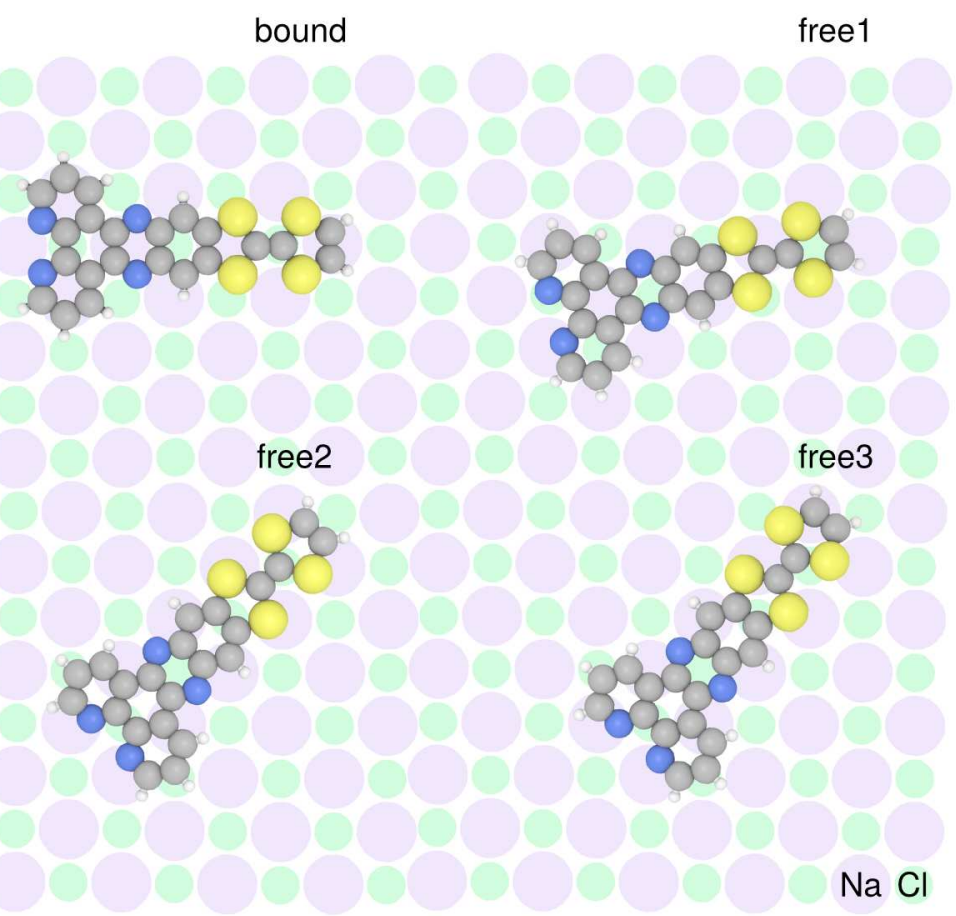

\section{b}
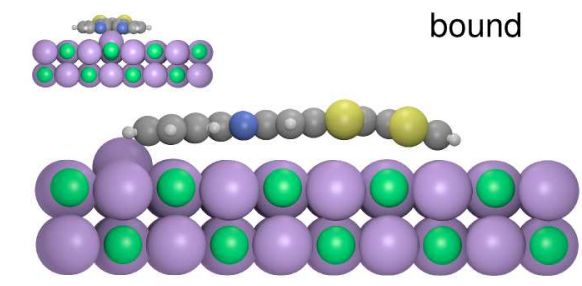

C
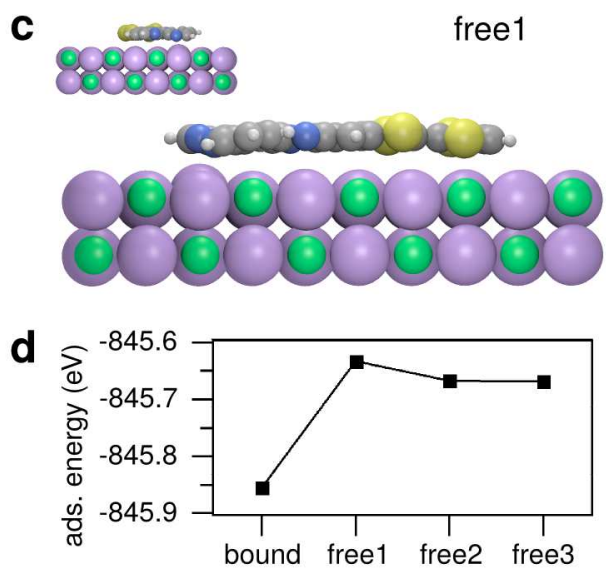

Figure 2: Simulated adsorption sites and geometries. a, Adsorption sites of TTFdppz calculated by DFT on $2 \mathrm{ML}$ of $\mathrm{NaCl}$ and observed in experiments. The experimentally determined adsorption sites are illustrated in Fig. S2. b,c, Side views of the relaxed bound molecule showing the pulling out of the $\mathrm{Na}^{+}$cation and of the free molecule depicted in Figure 1c; the insets are orthogonal back views. d, Calculated adsorption energies at different adsorption sites. For the representation of the $\mathrm{NaCl}$ layer the atomic radii are used. Color coding: grey $\mathrm{C}$, blue $\mathrm{N}$, yellow $\mathrm{S}$, pink $\mathrm{Na}$, green $\mathrm{Cl}$, white $\mathrm{H}$.

molecule observed at $V_{\mathrm{b}}=1.5 \mathrm{~V}$ exhibits two new lobes located at the nitrogen positions of the phenazine subunit (Fig. 3e) compared to the gap image (Fig. 3d). They can be attributed to the acceptor site and shows that a part of the LUMO is located at the dppz unit. However, there is a appreciable LUMO weight on the TTF side which clearly indicates a qualitative deviation from the calculated LUMO distribution of the gas phase molecule (Fig. S3d). This calculated LUMO distribution, centered on the dppz moiety, is in better agreement with the STM image at the LUMO resonance of the free molecule (Fig. 3h). Comparing the bound and free molecules, the location and shape of the LUMO resonances are quite different, as well as their energetic positions in the STS spectra. The onset of the LUMO resonance varies from $1 \mathrm{~V}$ for the bound to $1.5 \mathrm{~V}$ for the free molecule which also indicates that the electronic properties of the free molecules on the $\mathrm{NaCl}$ film are closer to 
those of the gas-phase molecules than for the bound ones. In addition, at negative bias the observed energetic shifts of the resonances indicate a different influence on the HOMO levels between bound and free adsorption sites. Since the HOMO is strongly localized on the TTF moiety, in a first approximation, the different adsorption geometries of the TTF unit are expected to be the dominant contribution for this feature.

The electronic properties are determined by the charge distribution in the molecule. Using force spectroscopy, i.e. measuring $\Delta f(V)$ which is proportional to the vertical force gradient, to resolve the local contact potential difference (LCPD) between molecule and tip, the intramolecular charge distribution is accessible in real-space as previously demonstrated with donor-acceptor molecules as well as other species. ${ }^{10,18,29,30}$ The LCPD contrast is known to vary with the tip-sample distance, particularly at close tip-sample distance, where the CO bending can produce complex contrasts. ${ }^{10}$ In our measurements, we used copper tips to characterize the LCPD contrasts which prevent probing at close-distance regimes compared to CO-terminated tips. In that case, i.e. at medium distance regime, compared to the molecule dimensions, the LCPD contrast represents the partial charge distribution of the DA molecules. Figure 4 shows the LCPD maps at constant tip height of the bound and free DA molecule, respectively. The LCPD corresponds to the voltage $V^{*}$ at the maximum frequency shift $\left(\Delta f^{*}\right)$ of the parabola fitted to the quadratic dependence of the frequency shift $\Delta f$ on the bias voltage $V .{ }^{10}$ The $\Delta f^{*}(x, y)$ maps reveal striking differences between the bound and free configurations (Fig. 4a,b). For bound molecules (Fig. 4a) $\Delta f^{*}$ exhibits a minimum at the edge of the TTF donor whereas, for free molecules, the $\Delta f^{*}$ drop extends over the whole molecule with an absolute minimum at the dppz location. This trend is also visible in the $\Delta f^{*}(x)$ profiles (Fig. 4c) extracted along the longitudinal axis of each molecule. Interestingly, the LCPD map of the bound molecule shows one region with a less negative LCPD compared to the surrounding $\mathrm{NaCl}$ layer which is attributed to local negative charges within the molecule (Fig. 4d). These negative charges are located between the phenazine subunit of the acceptor and the edge of the TTF donor. The most negative $\Delta f^{*}$ is detected at the same 
a
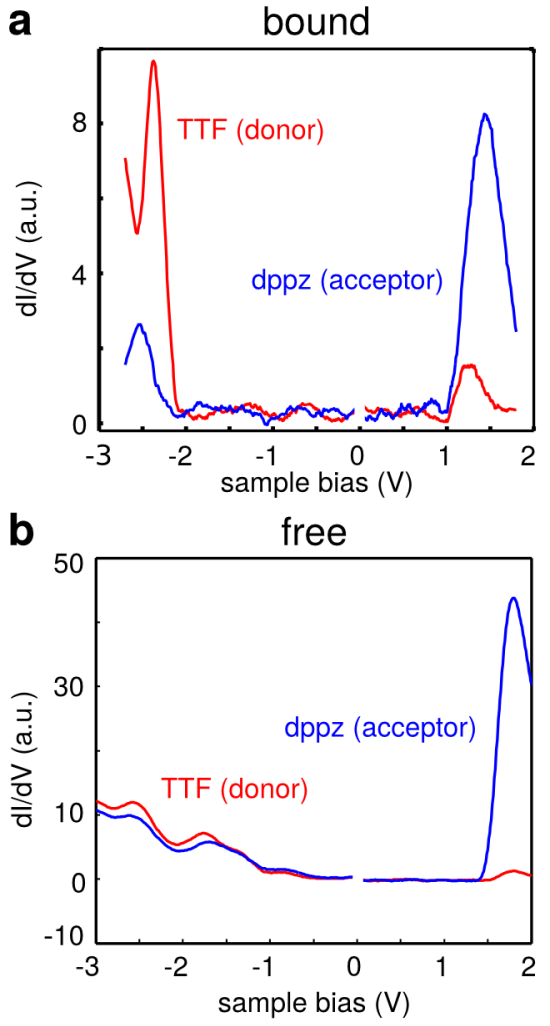

bound

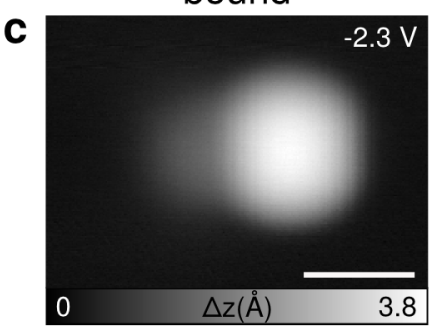

d

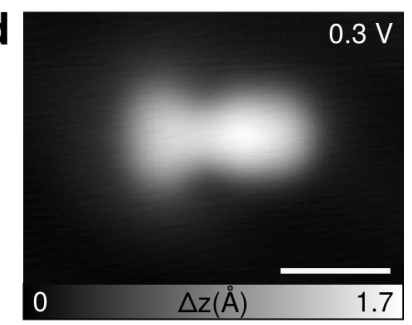

e

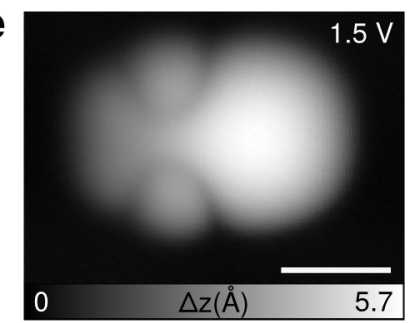

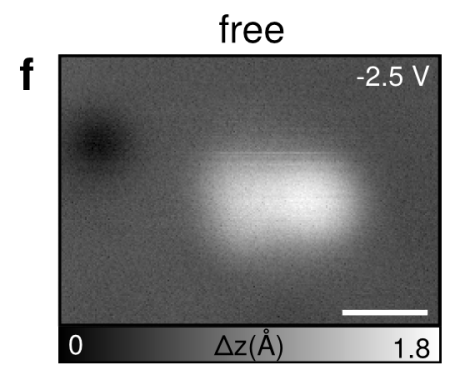

g

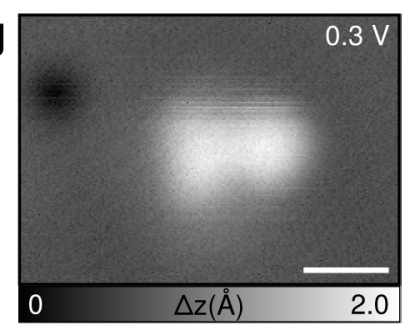

h

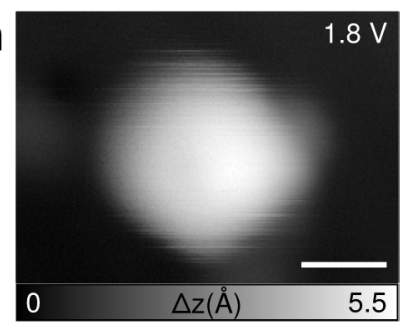

Figure 3: Energetic position and spatial distribution of the TTF-dppz' molecular orbitals. a,b, $\mathrm{d} I / \mathrm{d} V$ spectra of bound and free molecules, recorded on the donor (TTF) and acceptor (dppz) sides. c, STM constant current image of the bound molecule at the first resonance of negative sample bias $\left(I_{\mathrm{t}}=1 \mathrm{pA}, V_{\mathrm{b}}=-2.3 \mathrm{~V}\right)$, d, in the gap between the first negative and positive resonance $\left(I_{\mathrm{t}}=3 \mathrm{pA}, V_{\mathrm{b}}=0.3 \mathrm{~V}\right)$ and $\mathbf{e}$, at the first resonance of positive sample bias $\left(I_{\mathrm{t}}=1 \mathrm{pA}, V_{\mathrm{b}}=1.5 \mathrm{~V}\right)$. f, STM constant current image of the free molecule at $I_{\mathrm{t}}=1 \mathrm{pA}, V_{\mathrm{b}}=-2.5 \mathrm{~V}$, g, at $I_{\mathrm{t}}=1 \mathrm{pA}, V_{\mathrm{b}}=0.3 \mathrm{~V}$ and $\mathrm{h}$, at $I_{\mathrm{t}}=$ $1 \mathrm{pA}, V_{\mathrm{b}}=1.8 \mathrm{~V}$. The scale bars in $\mathbf{c}-\mathbf{h}$ correspond to $1 \mathrm{~nm}$. All STM/STS measurements were performed at $5 \mathrm{~K}$. See Fig. S3 for a comparison with the calculated distribution of the molecular orbitals in the gas phase.

position where the topographic maximum of the bound geometry is observed in AFM images and DFT calculations (Fig. 1b,d). For the free molecule (Fig. 4e), the LCPD also reveals a negative charge at this location. In contrast to the bound molecule, the least negative LCPD is clearly observed at the dppz moiety. This indicates that the acceptor side of the free molecule is more negatively charged compared to the surrounding $\mathrm{NaCl}$ whereas, for the bound molecule, the acceptor has the same charge relative to the surface. Furthermore, the LCPD at the TTF moiety of the free molecule is slightly below the background LCPD which suggests a positive charge at the donor (Fig. 4e). All the features are reproduced in 

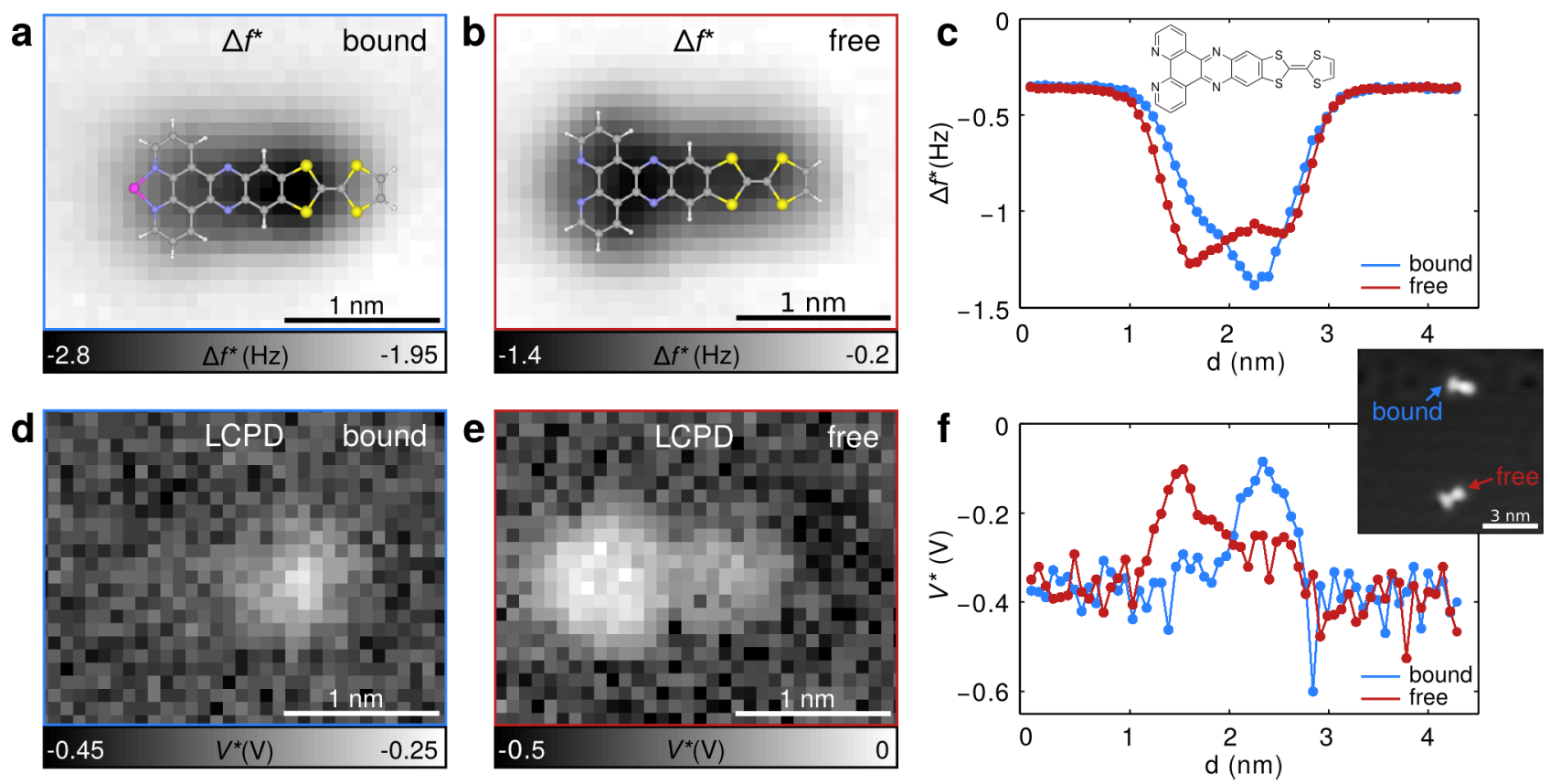

Figure 4: LCPD measurements of bound and free molecules. a, $\mathbf{d}, \Delta f^{*}$ and corresponding LCPD map of a bound molecule on $\mathrm{NaCl}(2 \mathrm{ML}) / \mathrm{Cu}(111)$. b, e, $\Delta f^{*}$ and LCPD maps of a free molecule adsorbed on $\mathrm{NaCl}(3 \mathrm{ML}) / \mathrm{Cu}(111)$. $\mathbf{c}, \mathbf{f}, \mathrm{LCPD}$ line scans along the molecular axis of bound and free molecules on $\mathrm{NaCl}(3 \mathrm{ML}) / \mathrm{Cu}(111)$, which are shown in the STM topography inset $\left(I_{\mathrm{t}}=3 \mathrm{pA}, V_{\mathrm{b}}=0.3 \mathrm{~V}\right)$ and measured with exactly the same $\mathrm{Cu}$ tip. For all LCPD measurements a Cu tip was used and the sample was kept at $5 \mathrm{~K}$.

the $V^{*}$ line scans (Fig. 4f) measured along the longitudinal axis of both molecules. The negative charge region is laterally shifted within the molecule. These observations demonstrate that bound and free molecules have different charge distributions which only depend on the adsorption site.

When the TTF-dppz molecule is aligned along the apolar axis of $\mathrm{NaCl}$, the bending of the dppz unit towards the surface is triggered by the coordination of the $\mathrm{N}$ atoms of the pyridyl units in dppz to the positively charged $\mathrm{Na}^{+}$cation in order to favor the formation of a chelate complex. Dppz is indeed known for its metal ion chelating property, mainly with ruthenium(II) and iron(II) ions ${ }^{31,32}$ but also with rare earth ions. ${ }^{33,34}$ Compared to transition metal ions, alkali metal ions exhibit weaker complexing abilities and chelation is usually essential to complex formation. ${ }^{35}$

A common notion is that the sodium ion and its higher congeners are not prone to 
bound
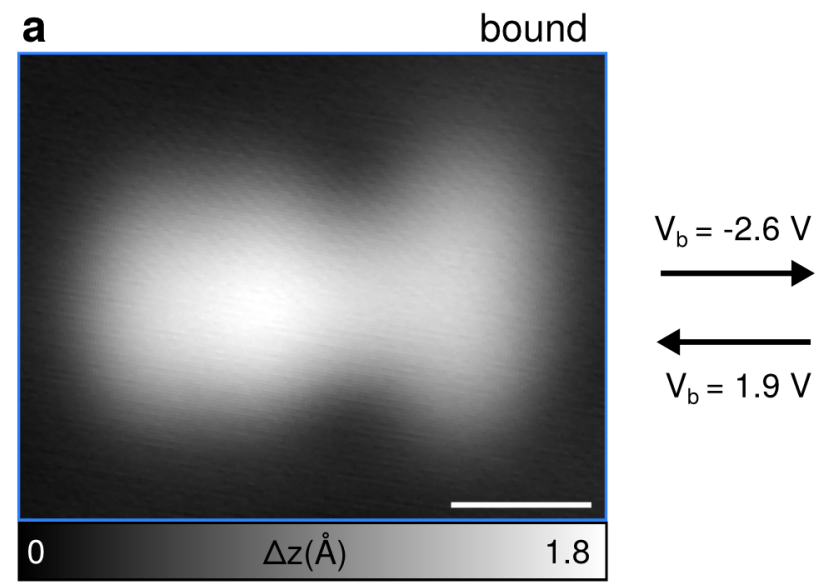

b
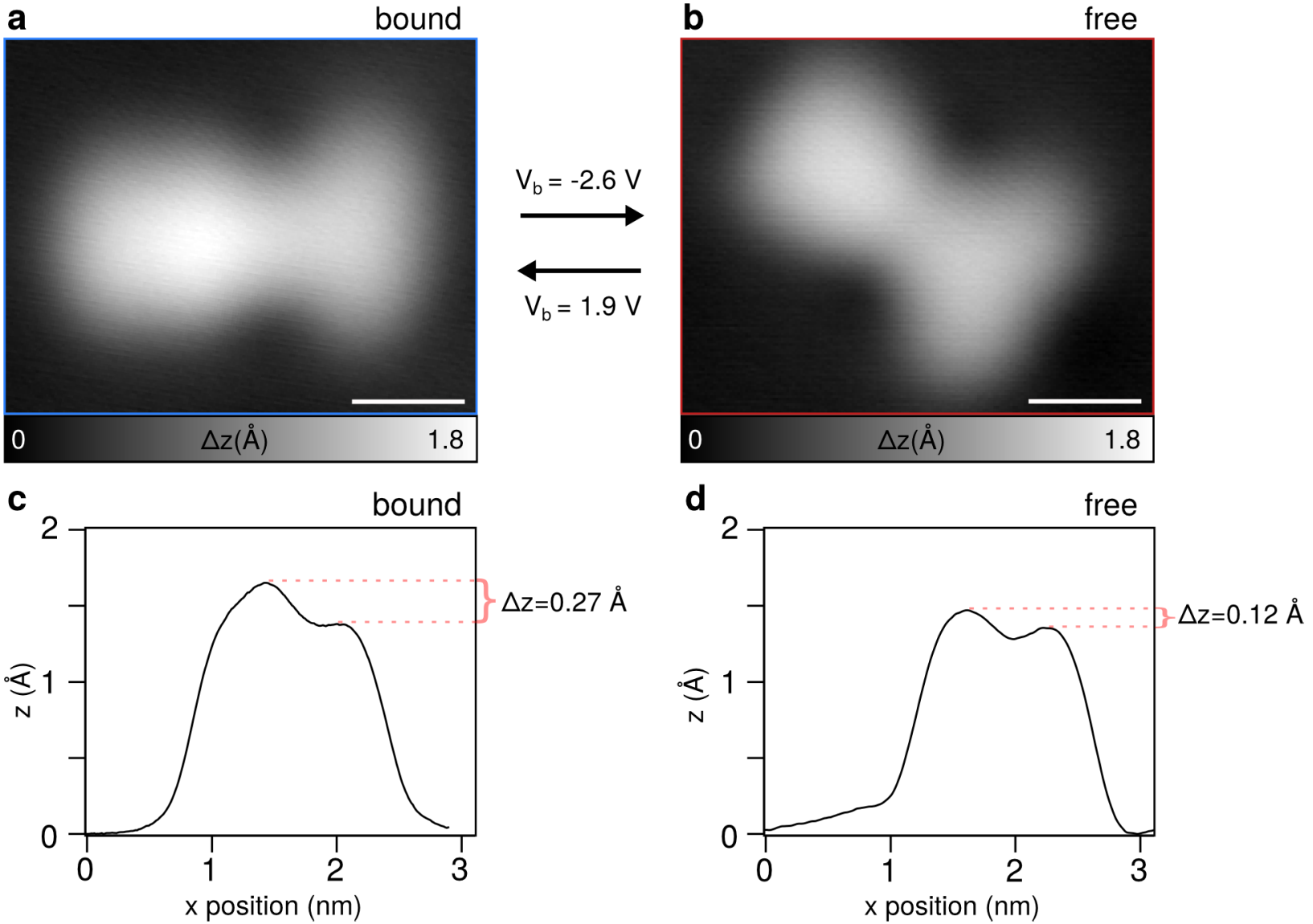

Figure 5: Switching between bound and free molecules. a,b, STM topography of bound and free molecule at $I_{\mathrm{t}}=3 \mathrm{pA}, V_{\mathrm{b}}=0.3 \mathrm{~V}$. Between the images the voltage was ramped until a jump in the current occured and the adsorption site changed. These voltage values are given next to the arrows. The tip was positioned above the topographic maximum of the molecule. The switching of the adsorption site could be done in both directions. The scale bars are $500 \mathrm{pm}$. c,d, Corresponding height profiles of the two molecules. Bound molecules show one distinct topographic maximum which forms the bridge shape geometry, whereas free molecules exhibit two local maxima.

generate coordination compounds and only the introduction of crown ethers and cryptands opened up different avenues for this field. From the viewpoint of Hard-Soft-Acid-Base theory, the coordination of sodium ions to ligands with oxygen donors clearly dominates over the coordination with nitrogen (soft donor) containing ligands, since $\mathrm{Na}^{+}$qualifies as a hard acid and oxygen as a hard donor. Consequently, there are only few reports on complexation of sodium ions with $\mathrm{N}^{\wedge} \mathrm{N}$ chelating ligands. ${ }^{36-38}$ Interestingly, the study of Qian et al. shows mono- and bidentate complexation modes for the coordination of sodium ions 
with phenanthroline-based ligands. ${ }^{37}$ For this rare case of a monodentate linkage, the Na-N coordination distances are in the range of $2.66 \AA$ to $2.73 \AA$, and the uncoordinated Na...N distances are larger than $3.19 \AA$. For the chelating $\mathrm{N}^{\wedge} \mathrm{N}$ coordination, the Na-N bond lengths range from $2.44 \AA$ to $2.46 \AA$, and the chelating N-Na-N bond angle amounts to $67.7^{\circ}$. Not unexpected, the above-mentioned bond distances are larger than those for a corresponding $\mathrm{O}^{\wedge} \mathrm{O}$ chelation mode of sodium ions, which typically shows values around $2.32 \AA .{ }^{39}$ From the DFT simulations (Fig.2), we extract the bond length between the Na and the pyridyl N atoms of $\sim 2.63 \AA$. Therefore, our measurements suggest the formation of a complex between $\mathrm{Na}^{+}$and $\mathrm{N}$ which is in the range of the referred Na-N coordination distance. The smaller bond length resulting from the bidentate complexation mode is likely not reached in our system since the $\mathrm{Na}^{+}$pulled out from the ionic film is still bound to the lattice.

As a result, the $\mathrm{Na}^{+}$alkali metal ion attracted by the lone pairs of the dppz $\mathrm{N}$ atoms forms a chelate complex which induces the intramolecular redistribution of charges. A reduction of negative charge at the acceptor side is indeed observed in the LCPD measurements of such complex (Fig. 4d,f) which suggests a partial charge transfer from the $\mathrm{N}$ atoms to the $\mathrm{Na}^{+}$altering the acceptor charge state. This interpretation is further confirmed in the STS measurements of the LUMO resonances shifting from the calculated LUMO distribution (Fig. S3d) to lower energies (Fig. 3a) as shown after complex formation. ${ }^{32}$ In contrast, the free molecules do not bind to a $\mathrm{Na}^{+}$cation and show a charge distribution (Fig. 4e) as predicted for DA molecules with a positive charge located at the donor region and a negative charge at the acceptor site. The simulated electrostatic potential of the free TTF-dppz (Fig. S3a) qualitatively agrees with the measured LCPD (Fig. 4e). For the bound type however, the variation of the electrostatic potential experimentally observed for the chelation is not as significant in the DFT calculations. Note that the charge around the $\mathrm{Na}^{+}$cation cannot be investigated directly in LCPD measurements since it is located close to the substrate and hence too far away from the tip to detect a LCPD difference at the position between 
molecule and substrate.

In order to demonstrate that only the adsorption sites affect the charge distribution and exclude the influence of possible defects of the $\mathrm{NaCl}$ thin layer, we conducted single-molecule manipulation experiments through inelastic electron tunneling (Fig. 5). ${ }^{40}$ We displaced a bound molecule to neighboring adsorption sites to induce the variation of electronic properties as described above. The investigation of the initial adsorption site confirmed that no defects are present below the bound molecule. Since the displaced molecule shows the typical STM topography of the free molecules, thus we further illustrate the interplay between the adsorption site and its molecular charge state. Switching in the opposite direction meaning from free to bound was also possible. These observations demonstrate that chelate complex formation of the $\mathrm{Na}^{+}-\mathrm{TTF}-\mathrm{dppz}$ can be controllably reversed by atomic manipulation.

\section{CONCLUSIONS}

We have demonstrated that interactions of a single DA molecule with the $\mathrm{NaCl}$ atomic lattice on $\mathrm{Cu}(111)$ result in the formation of a chelate complex with a single $\mathrm{Na}^{+}$cation. This chemical process depends on the positioning of the molecule with respect to the underlying substrate as characterized by combined high-resolution AFM measurements and DFT calculations. As a consequence of the $\mathrm{Na}^{+}$-molecule bond formation, the local alteration of the acceptor charge state results in the loss of the intrinsic DA molecule properties in the ground state. These findings highlight the interplay between the DA character of the molecule and the alternating charges of the insulating thin film at the atomic scale. Since the charge distribution in DA molecules in the ground state is strongly relevant to the properties in the excited state, our results are of great relevance for applications in optical energy conversion and molecular electronics where the functionality of specifically designed molecules must be maintained on surfaces. Interestingly, the complex formation can be triggered reversibly by 
voltage ramps thus enabling the design of a switchable donor-acceptor molecular compound controlled by coordination on an ionic thin film.

\section{METHODS}

STM/AFM experiments. The STM and AFM measurements were performed with an Omicron GmbH low temperature system equipped with a tuning fork in the Qplus configuration $^{41}\left(k \approx 1800 \mathrm{~N} / \mathrm{m}, f_{\text {res }}=25 \mathrm{kHz}\right)$ and operated with Nanonis controllers RC5. For all measurements the sample was kept at $5 \mathrm{~K}$. The amplitude of the tuning fork oscillation was set to a value between 50 - 100 pm. Scanning tunneling spectroscopy (STS) data was acquired with the lock-in technique. The bias modulation amplitude was $10 \mathrm{mV}$ at a frequency of $520 \mathrm{~Hz}$. The bias voltage is given by the potential of the sample referred to the tip. The STM/AFM tip consists of a tungsten wire that is gently indented into the clean $\mathrm{Cu}(111)$ surface several times to obtain a $\mathrm{Cu}$ tip. All STM images were recorded with a $\mathrm{Cu}$ tip, unless a different termination is explicitly stated. CO tip terminations were done by picking up single $\mathrm{CO}$ molecules adsorbed on the $\mathrm{NaCl}$ film. For the LCPD mapping, a measurement grid was layed over the molecule. At each point of the grid a bias sweep was performed and the frequency shift was detected. Between the bias sweeps the position was adjusted by atom tracking. ${ }^{42}$

Sample preparation. The $\mathrm{Cu}(111)$ surface was prepared in ultra high vacuum by sputtering and annealing cycles. Two to three monolayers of sodium chloride (NaCl, purchased from Sigma-Aldrich $\mathrm{GmbH}$ ) were then deposited on the surface at room temperature. Tetrathiafulvalene-fused dipyridophenazine (TTF-dppz) molecules were thermally evaporated onto the cold substrate $\left(T_{\text {sample }}<10 \mathrm{~K}\right)$ to obtain single-molecules.

DFT Calculations Calculations were performed using the projector augumented planewave $^{43}$ method and the PBE functional ${ }^{44}$ as implemented in the VASP 5.3 code. ${ }^{45}$ Planewave cutoffs were set to $400 \mathrm{eV}$ for wave-functions and $644 \mathrm{eV}$ for potential. The rectangular 
simulation box of $\mathrm{x}, \mathrm{y}, \mathrm{z}$ dimensions $28.2 \mathrm{x} 22.56 \mathrm{x} 20.00 \AA$ corresponds to a $4 \mathrm{x} 5$ super-cell containing a $\mathrm{NaCl}$ (100) slab composed of 2 layers. Only the $\Gamma$ point was used for $\mathrm{k}$-space sampling. Convergence criterion was set to $10^{-6} \mathrm{eV} /$ step for electronic and $10^{-5} \mathrm{eV} / \mathrm{step}$ for ionic relaxation.

Synthesis of 2-(1,3-dithiol-2-ylidene)-[1,3]benzodithiole-5,6-diamine (compound 2): Under $\mathrm{N}_{2}$ protection, 6-(1,3-dithiol-2-ylidene)-[1,3] dithiolo[4,5-f]-2,1,3-benzothiadiazole ${ }^{46}$ (156 mg, $0.5 \mathrm{mmol})$ was dissolved in dry THF $(10 \mathrm{~mL})$. Then Lithium aluminum hydride (1.25 mL, $1 \mathrm{M}$ in THF, $1.25 \mathrm{mmol}$ ) was added dropwise to the reaction mixture over a period of $30 \mathrm{~min}$ at $0^{\circ} \mathrm{C}$. The reaction mixture was then stirred for 30 min under nitrogen. Afterwards it was slowly quenched by saturated aqueous $\mathrm{NH}_{4} \mathrm{Cl}(1 \mathrm{~mL})$ at $0^{\circ} \mathrm{C}$. The resulting precipitate was collected and washed by water and $\mathrm{Et}_{2} \mathrm{O}$ to give analytically pure product as a light yellow solid. Yield: $131 \mathrm{mg}(91 \%) ;{ }^{1} \mathrm{H}$ NMR $\left(300 \mathrm{MHz}, \mathrm{DMSO}-\mathrm{d}_{6}\right) \delta=6.72(\mathrm{~s}, 2 \mathrm{H})$, $6.54(\mathrm{~s}, 2 \mathrm{H}), 4.68(\mathrm{~s}, 4 \mathrm{H}) ;{ }^{13} \mathrm{C} \operatorname{NMR}\left(75.5 \mathrm{MHz}, \mathrm{THF}-\mathrm{d}_{8}\right): \delta=134.8,121.6,119.8,111.5$, 107.6, 107.5 ppm; HRMS (ESI): m/z calcd for $\mathrm{C}_{10} \mathrm{H}_{8} \mathrm{~N}_{2} \mathrm{~S}_{4}$ : 283.9565; found 283.9556 .

Synthesis of 2-(1,3-dithiol-2-ylidene)-[1,3]dithiolo[4,5-i]dipyrido[3,2-a:2,3-c]phenazine (TTF-dppz): A solution of compound $2(57 \mathrm{mg}, 0.2 \mathrm{mmol})$ and 1,10-phenanthroline-5,6dione (41 mg, $0.2 \mathrm{mmol})$ in ethanol $(40 \mathrm{~mL})$ was refluxed for $3 \mathrm{~h}$ under $\mathrm{N}_{2}$. After filtration, the precipitate was washed by $\mathrm{CH}_{2} \mathrm{Cl}_{2}$ and purified by recrystallization in toluene to give analytically pure TTF-dppz as a deep blue powder (50 mg, 55\%). ${ }^{1} \mathrm{H} \mathrm{NMR} \mathrm{(300} \mathrm{MHz,}$ $\left.\operatorname{DMSO}_{-} \mathrm{d}_{6}\right) \delta=9.48(\mathrm{~d}, \mathrm{~J}=8.1,2 \mathrm{H}), 9.21(\mathrm{~d}, \mathrm{~J}=4.4 \mathrm{~Hz}, 2 \mathrm{H}), 8.40(\mathrm{~s}, 2 \mathrm{H}), 7.92(\mathrm{dd}, \mathrm{J}=$ 8.1, $4.4 \mathrm{~Hz}, 2 \mathrm{H}), 6.82(\mathrm{~s}, 2 \mathrm{H}) ;{ }^{13} \mathrm{C} \mathrm{NMR}$ is not available due to its limited solubility. HRMS (ESI): $\mathrm{m} / \mathrm{z}$ calcd for $\mathrm{C}_{22} \mathrm{H}_{10} \mathrm{~N}_{4} \mathrm{~S}_{4}$ : 457.9861; found 457.9866. Further details on the synthesis of TTF-dppz can be found in the Supplementary Information. 


\title{
AUTHOR INFORMATIONS
}

\author{
Corresponding Authors \\ *E-mails: tobias.meier@unibas.ch, thilo.glatzel@unibas.ch
}

\begin{abstract}
Author Contributions
E.M., S.D., T.G. and R.P. planned the experiments. T.M. performed the STM/AFM experiments, processed the data and wrote the manuscript. P.H. and P.J. performed the DFT calculations. Y.G., X.L., S.X.L. and S.D. synthesized the molecular compounds. All coauthors commented the data and contributed to the manuscript.
\end{abstract}

\section{ORCID}

Tobias Meier 0000-0003-0606-5131

\section{ACKNOWLEDGEMENTS}

This work was supported in by the Swiss National Science Foundation (SNF), the Swiss Nanoscience Institue (SNI), the EU-Cost-Actions MP1303 and MP1403, the European Commission (EC) FP7 ITN 'MOLESCO' (project no. 606728) and by the Japan Science and Technology Agency (JST) 'Precursory Research for Embryonic Science and Technology (PRESTO)' for a project of 'Molecular technology and creation of new functions', by Japan Society for the Promotion of Science (JSPS) KAKENHI (grant no 15K21765).

P.H. and P.J. acknowledge support of the Czech Science Foundation (grant no. 14-374527G). 


\section{ASSOCIATED CONTENT}

\section{Supporting Information Available}

Further details concerning experiments, simulations and synthesis can be found in the Supplementary material section. This material is available free of charge via the Internet at http://pubs.acs.org/.

\section{REFERENCES}

(1) Jia, C.; Liu, S.-X.; Tanner, C.; Leiggener, C.; Neels, A.; Sanguinet, L.; Levillain, E.; Leutwyler, S.; Hauser, A.; Decurtins, S. An Experimental and Computational Study on Intramolecular Charge Transfer: A Tetrathiafulvalene-Fused Dipyridophenazine Molecule. Chem. - Eur. J. 2007, 13, 3804-3812.

(2) Aviram, A.; Ratner M. A. Molecular Rectifiers. Chem. Phys. Lett. 1974, 29, 277-283.

(3) Carroll, R. L.; Gorman, C. B. The Genesis of Molecular Electronics. Angew. Chem., Int. Ed. 2002, 41, 4378-440.

(4) Jurow M.; Schuckmann, A. E.; Batteas, J. D.; Drain, C. M. Porphyrins as Molecular Electronic Components of Functional Devices. Coord. Chem. Rev. 2010, 254, 22972310.

(5) Metzger, R. M., Single-Molecule Electronics. In Supramolecular Chemistry: From Molecules to Nanomaterials; Steed, J. W., Gale, P. A., Eds.; Wiley: New York, 2012.

(6) Bergkamp, J.; Decurtins, S.; Liu, S.-X. Current Advances in Fused Tetrathiafulvalene Donor-Acceptor Systems. Chem. Soc. Rev. 2015, 44, 863-874.

(7) Amacher, A.; Yi, C.; Yang, J.; Bircher, M. P.; Fu, Y.; Cascella, M.; Grätzel, M.; 
Decurtins, S.; Liu, S.-X. A Quinoxaline-Fused Tetrathiafulvalene-Based Sensitizer for Efficient Dye-Sensitized Solar Cells. Chem. Commun. 2014, 50, 6540-6542.

(8) Metzger, R. M. Unimolecular Electrical Rectifiers. Chem. Rev. 2003, 103, 3803-3834.

(9) Lörtscher, E.; Gotsmann, B.; Lee, Y.; Yu, L.; Rettner, C.; Riel, H. Transport Properties of a Single-Molecule Diode. ACS Nano 2012, 6, 4931-4939.

(10) Schuler, B.; Liu, S.-X.; Geng, Y.; Decurtins, S.; Meyer, G.; Gross, L. Contrast Formation in Kelvin Probe Force Microscopy of Single $\pi$-Conjugated Molecules. Nano Lett. 2014, 14, 3342-3346.

(11) Wu, S. W.; Ogawa, N.; Nazin, G.V.; Ho, W. Conductance Hysteresis and Switching in a Single-Molecule Junction. J. Phys. Chem. C 2008, 112, 5241-5244.

(12) Pavliček, N.; Fleury, B.; Neu, M.; Niedenführ, J.; Herranz-Lancho, C.; Ruben, M.; Repp, J. Atomic Force Microscopy Reveals Bistable Configurations of Dibenzo[a,h]thianthrene and Their Interconversion Pathway. Phys. Rev. Lett. 2012, $108,086101$.

(13) De Oteyza, D. G.; Gorman, P.; Chen, Y.-C.; Wickenburg, S.; Riss, A.; Mowbray, D.J.; Etkin, G.; Pedramrazi, Z.; Tsai, H.-Z.; Rubio, A.; Crommie, M. F.; Fischer, F. R. Direct Imaging of Covalent Bond Structure in Single-Molecule Chemical Reactions. Science 2013, 340, 1434.

(14) Schuler, B.; Fatayer, S.; Mohn, F.; Moll, N.; Pavliček, N.; Meyer, G.; Peña, D.; Gross, L. Reversible Bergman Cyclization by Atomic Manipulation. Nat. Chem. 2016, 8, 220224.

(15) Pavliček, N.; Schuler, B.; Collazos, S.; Moll, N.; Pérez, D.; Guitán, E.; Meyer, G.; Peña, D.; Gross, L. On-Surface Generation and Imaging of Arynes by Atomic Force Microscopy. Nat. Chem. 2015, 7, 623-628. 
(16) Kawai, S.; Haapasilta, V.; Lindner, D. B.; Tahara, K.; Spijker, P.; Buitendijk, J. A.; Pawlak, R.; Meier, T.; Tobe, Y.; Foster, A. S.; Meyer, E. Thermal Control of Sequential On-Surface Transformation of a Hydrocarbon Molecule on a Copper Surface. Nat. Commun. 2016, 7, 12711.

(17) Kocić, N.; Liu, X.; Chen, S.; Decurtins, S.; Krejčí, O.; Jelínek, P.; Repp, J.; Liu, S.-X. Control of Reactivity and Regioselectivity for On-Surface Dehydrogenative Aryl-Aryl Bond Formation. J. Am. Chem. Soc. 2016, 138, 5585-5593.

(18) Mohn, F.; Gross, L.; Moll, N.; Meyer, G. Imaging the Charge Distribution within a Single Molecule. Nature 2012, 7, 227-231.

(19) Wu, S. W.; Nazin, G. V.; Chen, X.; Qiu X. H.; Ho, W. Control of Relative Tunneling Rates in Single Molecule Bipolar Electron Transport. Phys. Rev. Lett. 2004, 93, 236802.

(20) Repp, J.; Meyer, G.; Stojković, S. M.; Gourdon, A.; Joachim C. Molecules on Insulating Films: Scanning-Tunneling Microscopy Imaging of Individual Molecular Orbitals. Phys. Rev. Lett. 2005, 94, 026803.

(21) Leoni, T.; Guillermet, O.; Walch, H.; Langlais, V.; Scheuermann, A.; Bonvoisin, J.; Gauthier, S. Controlling the Charge State of a Single Redox Molecular Switch. Phys. Rev. Lett. 2011, 106, 216103.

(22) Repp, J.; Meyer, G.; Olsson, F. E.; Persson, M. Controlling the Charge State of Individual Gold Adatoms. Science 2004, 305, 493-495.

(23) Gross, L.; Mohn, F.; Liljeroth, P.; Repp, J.; Giessibl, F. J.; Meyer, G. Measuring the Charge State of an Adatom with Noncontact Atomic Force Microscopy. Science 2009, 324, 1428-1431.

(24) Olsson, F. E.; Paavilainen, S.; Persson, M.; Repp, J.; Meyer, G. Multiple Charge States of Ag Atoms on Ultrathin $\mathrm{NaCl}$ Films. Phys. Rev. Lett. 2007, 98, 176803. 
(25) Such, B.; Trevethan, T.; Glatzel, T.; Kawai, S.; Zimmerli, L.; Meyer, E.; Shluger, A. L.; Amijs, C. H. M.; De Mendoza, P.; Echavarren, A. M. Functionalized Truxenes: Adsorption and Diffusion of Single Molecules on the $\operatorname{KBr}(001)$ Surface. ACS Nano 2010, 4, 3429-3439.

(26) Schuler, B.; Liu, W.; Tkatchenko, A.; Moll, N.; Meyer, G.; Mistry, A.; Fox, D.; Gross, L. Adsorption Geometry Determination of Single Molecules by Atomic Force Microscopy. Phys. Rev. Lett. 2013, 111, 106103.

(27) Hapala, P.; Temirov, R.; Tautz, F. S.; Jelínek, P. Origin of High-Resolution IETS-STM Images of Organic Molecules with Functionalized Tips. Phys. Rev. Lett. 2014, 113, 226101.

(28) Tao, C.; Sun, J.; Zhang, X.; Yamachika, R.; Wegner, D.; Bahri, Y.; Samsonidze G.; Cohen, M. L.; Louie, S. G.; Tilley, T. D.; Segalman, R. A.; Crommie M. F. Spatial Resolution of a Type II Heterojunction in a Single Bipolar Molecule. Nano Lett. 2009, 9, 3963-3967.

(29) Kawai, S.; Sadeghi, A.; Feng, X.; Lifen, P.; Pawlak, R.; Glatzel, T.; Willand, A.; Orita, A.; Otera, J.; Goedecker, S.; Meyer, E. Obtaining Detailed Structural Information about Supramolecular Systems on Surfaces by Combining High-Resolution Force Microscopy with Ab Initio Calculations. ACS Nano 2013, 7, 9098-9105.

(30) Moll, N.; Schuler, B.; Kawai, S.; Xu, F.; Peng, L; Orita, A.; Otera, J.; Curioni, A.; Neu, M.; Repp, J.; Meyer, G.; Gross, L. Image Distortions of a Partially Fluorinated Hydrocarbon Molecule in Atomic Force Microscopy with Carbon Monoxide Terminated Tips. Nano Lett. 2014, 14, 6127-6131.

(31) Goze, C.; Leiggener, C.; Liu, S.-X.; Sanguinet, L.; Levillain, E.; Hauser, A.; Decurtins, S. Fused Donor-Acceptor Ligands in RuII Chemistry: Synthesis, Electrochemistry and Spectroscopy of $\left[\mathrm{Ru}(\mathrm{bpy})_{3-n}(\mathrm{TTF}-\mathrm{dppz})_{n}\right]\left(\mathrm{PF}_{6}\right)_{2}$. ChemPhysChem 2007, 8, 1504-1512. 
(32) Dupont, N.; Ran, Y.-F.; Liu, S.-X.; Grilj, J.; Vauthey, E.; Decurtins, S.; Hauser, A. A DonorAcceptor Tetrathiafulvalene Ligand Complexed to Iron(II): Synthesis, Electrochemistry, and Spectroscopy of $\left[\mathrm{Fe}(\mathrm{phen})_{2}(\mathrm{TTF}-\mathrm{dppz})\right]\left(\mathrm{PF}_{6}\right)_{2}$. Inorg. Chem. 2013, 52, $306-312$.

(33) Lapadula, G.; Trummer, D.; Conley, M. P.; Steinmann, M.; Ran, Y.-F.; Brasselet, S.; Guyot, Y.; Maury, O.; Decurtins, S.; Liu, S.-X.; Copéret, C. One-Photon NearInfrared Sensitization of Well-Defined Yb(III) Surface Complexes for NIR-to-NIR Single Nanoparticle Imaging. Chem. Mater. 2015, 27, 2033-2039.

(34) Pointillart, F.; Jung, J.; Berraud-Pache, R.; Le Guennic, B.; Dorcet, V.; Golhen, S.; Cador, O.; Maury, O.; Guyot, Y.; Decurtins, S.; Liu, S.-X., Ouahab, L. Luminescence and Single-Molecule Magnet Behavior in Lanthanide Complexes Involving a Tetrathiafulvalene-Fused Dipyridophenazine Ligand. Inorg. Chem. 2015, 54, 53845397.

(35) Poonia, N. S.; Kumar, G. C.; Jayakumar, A.; Bagdi, P.; Bajaj, A.V. Coordination Chemistry of Alkali and Alkaline Earth Cations. Chem. Rev. 1979, 43, 2159-2164.

(36) Hughes, D. L. Alkali-metal complexes. Part VII. Crystal and Molecular Structures of the o-Nitrophenolatobis(1,10-Phenanthroline) Complexes of Sodium and Rubidium. J. Chem. Soc., Dalton Trans. 1973, 2347-2354..

(37) Qian, H.-F.; Liu, Y.; Tao, T.; Gu, K.-H.; Yin, G.; Huang, W. Four Sodium Tetrafluoroborate Directed Supramolecular Networks Having 3- and 3,8-Disubstituted 1,10Phenanthroline Ligands. Inorg. Chim. Acta 2013, 405, 1-8.

(38) Schmidt, E.; Hourdakis, A.; Popov, A. I. Multinuclear NMR Study of 2,2'-Bipyridine Complexes with Alkali Cations in Nonaqueous Solvents. Inorg. Chim. Acta 1981, 52, $91-95$. 
(39) Decurtins, S.; Schmalle, H. W.; Schneuwly, P.; Ensling, J.; Gütlich, P. A Concept for the Synthesis of 3-dimensional Homo- and Bimetallic Oxalate-Bridged Networks $\left[\mathrm{M}_{2}(\mathrm{ox})_{3}\right]_{\mathrm{n}}$. Structural, Mössbauer, and Magnetic Studies in the Field of MolecularBased Magnets. J. Am. Chem. Soc. 1994, 116, 9521-9528.

(40) Swart, I.; Sonnleitner, T.; Niedenführ, J.; Repp, J. Controlled Lateral Manipulation of Molecules on Insulating Films by STM. Nano Lett. 2012, 12, 1070-1074.

(41) Giessibl, F. J. Advances in Atomic Force Microscopy. Rev. Mod. Phys. 2003, 75, 949983.

(42) Kawai, S.; Glatzel, T.; Koch, S.; Baratoff, A.; Meyer, E. Interaction-Induced Atomic Displacements Revealed by Drift-Corrected Dynamic Force Spectroscopy. Phys. Rev. B 2011, 83, 035421 .

(43) Kresse, G.; Joubert, D. From Ultrasoft Pseudopotentials to the Projector Augmented Wave Method, Phys. Rev. B 1999, 59, 1758-1775.

(44) Perdew, J. P.; Burke, K.; Ernzerhof, M. Generalized Gradient Approximation Made Simple. Phys. Rev. Lett., 1996, 77, 3865-3868.

(45) Kresse, G.; Furthmüller, J. Efficient Iterative Schemes for Ab Initio Total-Energy Calculations Using a Plane-Wave Basis Set. Phys. Rev. B 1996, 54, 11169-11186.

(46) Geng, Y.; Pfattner, R.; Campos, A.; Hauser, J.; Laukhin, V.; Puigdollers, J.; Veciana, J.; Mas-Torrent, M.; Rovira, C.; Decurtins, S.; Liu, S.-X. A Compact Tetrathiafulvalene-Benzothiadiazole Dyad and Its Highly Symmetrical Charge-Transfer Salt: Ordered Donor $\pi$-Stacks Closely Bound to Their Acceptors. Chem. - Eur. J. 2014, 20, 7136-7143. 


\section{Graphical TOC Entry}

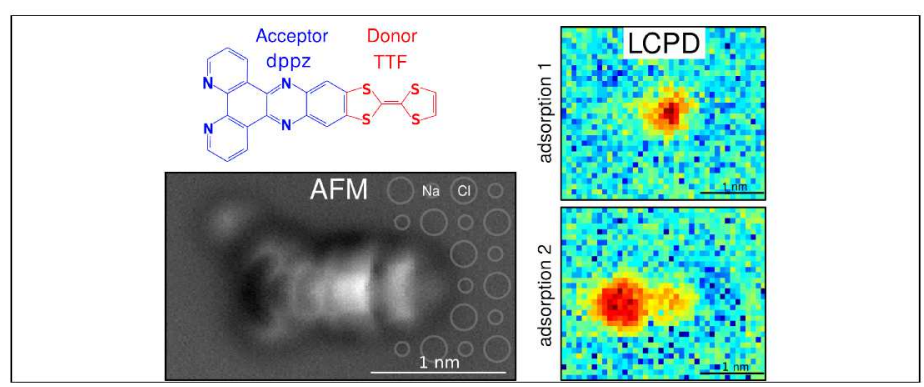

